Case Report

\title{
A Rare Presentation of Actinic Keratosis Affecting the Tarsal Conjunctiva and Review of the Literature
}

\author{
Selina Khan $(\mathbb{D})$ and Melanie Chak $(i)$ \\ Ophthalmology Department, Great Western Hospital, Swindon, UK \\ Correspondence should be addressed to Selina Khan; sk7105@my.bristol.ac.uk
}

Received 31 October 2017; Accepted 15 January 2018; Published 12 February 2018

Academic Editor: Alexander A. Bialasiewicz

Copyright (C) 2018 Selina Khan and Melanie Chak. This is an open access article distributed under the Creative Commons Attribution License, which permits unrestricted use, distribution, and reproduction in any medium, provided the original work is properly cited.

\begin{abstract}
We report an unusual case of actinic keratosis (AK) of the tarsal conjunctiva in a 63-year-old man. Examination revealed a crusty, leukoplakic lesion prone to bleeding on the tarsal conjunctiva of the right upper eyelid. This was treated by surgical excisional biopsy. At 1-year follow-up, there was no evidence of recurrence and the surgical site was completely healed without conjunctival scarring. Current opinion cites excessive exposure to ultraviolet (UV) radiation, in particular UV-B in sunlight, as the causative agent in developing AK. In the case we present, the tarsal conjunctiva is an unusual place for actinic keratosis due to the lack of direct UV-light exposure. The key learning points are to evert the upper eyelid during examination especially if the lesion involves the eyelid margin and, secondly, to ensure risk factors are addressed during the history.
\end{abstract}

\section{Introduction}

Leukoplakia describes the whitish appearance of squamous epithelium after metaplastic keratinisation has occurred. Typically it affects nonkeratinised mucous membranes such as the oral cavity and larynx. There have been a few reports of leukoplakia of the conjunctiva and cornea. In the case we report, the leukoplakia was due to actinic keratosis (AK) also known as solar keratosis, of the tarsal conjunctiva and eyelid margin. The tarsal conjunctiva is an unusual presentation due to lack of direct UV radiation exposure.

\section{Case Presentation}

A 63-year-old, Caucasian man presented to outpatients' clinic with a crusty, bleeding, right upper eyelid lesion. This had gradually appeared over the past 12 months. Examination revealed two distinct lesions of leukoplakia on the right upper eyelid margin, extending onto the tarsal conjunctiva found on eye lid eversion and measuring $5 \mathrm{~mm}$ horizontally (Figure 1). Slit lamp biomicroscopy after $2 \%$ fluorescein staining showed multiple punctate epithelial erosions localised to the superior corneal surface. In 2013, he underwent surgical excision of a lesion to the right upper eyelid in a similar topography but limited to the skin. This was diagnosed as actinic keratosis on histological examination. He reported no significant history of prolonged sunlight exposure throughout his life. He had no history of skin cancer. He was not immunosuppressed.

He was treated for the new lesions with a surgical excisional biopsy of the eyelid margin and tarsal conjunctiva under local anaesthetic. Histological examination confirmed features consistent with actinic keratosis on the eyelid margin and tarsal conjunctiva (Figures 2, 3(a), and 3(b)).

At 1-month follow-up after surgical excision, examination revealed complete resolution of the punctate epithelial erosions to the cornea and a healthy superior tarsal conjunctiva to both eyes. The cutaneous margin of the right eye, which was included in the biopsy, had fully healed without any complications. At 1 year follow-up, there was no evidence of recurrence.

\section{Discussion}

Actinic keratosis (AK) is defined as the neoplastic transformation and proliferation of keratinocytes within the epidermis without breaching the basement membrane [1]. Excessive exposure to UV radiation, primarily UV-B in 


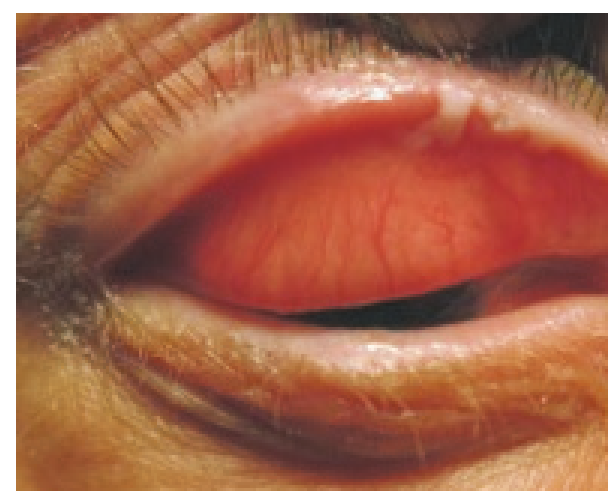

FIGURE 1: Photograph of the right-upper eyelid leukoplakia prior to excisional biopsy. Leukoplakia is present on eyelid margin and tarsal conjunctiva.

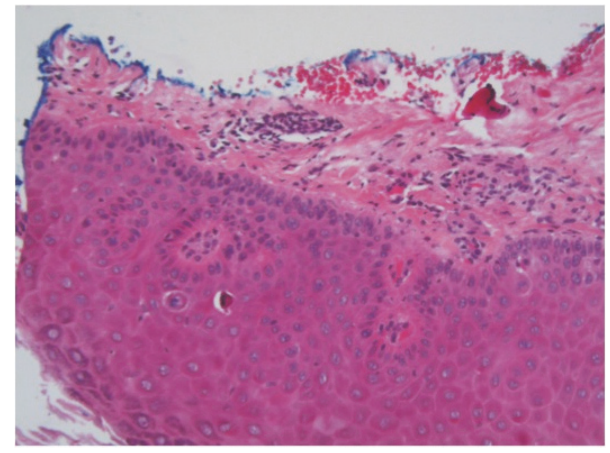

FIGURE 2: Histology specimen of the tarsal conjunctiva and eyelid margin. Marked hyperkeratosis and parakeratosis of the eyelid margin with extension of this cell atypia into the tarsal conjunctiva. Haematoxylin and Eosin, 100x magnification.

sunlight, induces these changes which typically affect sunexposed areas [2]. It is the chronic UV-B light exposure which results in damage to DNA and its repair mechanisms, specifically inducing mutations to the p53 gene, a key regulator of the cell cycle and tumourogenesis [3]. In the case of AK, transformation into squamous cell carcinoma follows the 3 stages of photocarcinogenesis: initiation, that is, UVlight exposure, promotion (clonal expansion of metaplastic cells), and conversion through further genetic mutations into squamous cell carcinoma [4].

In addition to cumulative sun exposure, other risk factors for AK include fair skin, advanced age, exposure to carcinogens, prolonged immunosuppression, and genetic conditions affecting DNA repair mechanisms such as xeroderma pigmentosum [5]. Most commonly AK is found on sun-exposed areas such as the hands, forearms, and face.

Clinically it is typically characterised by multiple, erythematous, nodular plaques ranging between 1 and $10 \mathrm{~mm}$, unlike our case which presented with a leukoplakic lesion. The mainstay of cutaneous actinic keratosis treatment is complete excision biopsy. Alternatively topical chemotherapeutic agents such as 5-fluorouracil (5-FU) cream applied twice daily for 2-3 weeks and recently topical imiquimod 3 times per week for 16 weeks have been effective $[1,6,7]$.

Reports of AK affecting the conjunctiva have been limited to the bulbar conjunctiva, with none reporting the management of tarsal involvement. These cases have been treated primarily with excisional biopsy and more recently with a combination of chemotherapeutic agents [8-11]. Rowlands et al. are the first group to successfully attempt complete eradication of bulbar conjunctival AK with $5 \%$ imiquimod after recurrence with cryotherapy and Interferon Alpha$2 \mathrm{~b}$ [8]. Previous studies described no recurrence of actinic keratosis affecting the limbal conjunctiva when treated with excisional biopsy at 18 months' follow-up $[9,10]$.

Success with excisional biopsy for cutaneous AK is welldocumented in the literature, less so but increasingly for bulbar conjunctival AK. Conservative therapy with topical agents with or without surgical excision remains an area to be further explored especially for multiple lesions.

A recent multicentre phase 2 trial reported complete clearance in $35.6 \%$ of individuals with $\mathrm{AK}$ to the face and chest with a once-daily for 3 days application regime of ingenol disoxate gel [12]. This is comparable to complete clearance rates for 5-FU, imiquimod, and diclofenac sodium $3 \%$ gel $[13,14]$. Ingenol disoxate is a novel derivative to the ingenol mebutate molecule. This newer topical therapy induces rapid cell death and is able to exert its action in the dermis directly and indirectly by stimulating an immunogenic cytokine response. Consequently, it only requires a 23 -day course of treatment which may increase compliance to treatment in comparison to alternatives such as 5-FU or imiquimod which require treatment for a minimum of 2 weeks. As of yet, there are no reports of its use in an ophthalmic context.

Treatment of periocular AK with topical diclofenac sodium 3\% gel, used twice daily for up to 4 months, had resulted in complete resolution initially. However recurrence in $50 \%$ occurred in this study and further treatment with surgical excision was required in one case and adjuvant cryotherapy in another [15]. A case series of 13 patients with periocular AK treated with 5-FU for 14 days, twice a day, 9 of which with eye margin involvement, reported complete resolution at final follow-up. Five patients required a second course of 5-FU with minimal adverse reactions reported [6].

Our case report demonstrates successful, minimally destructive treatment of tarsal conjunctiva and eyelid margin AK with surgical excision without recurrence at 12 months. It also emphasises the importance of eyelid eversion for all patients with lesions involving the eyelid especially if adjacent to the margin. This is to check for the extension of the lesion and to allow for planning of appropriate management. Specifically, the tarsal conjunctiva rests adjacent to the cornea and when administering treatment to this region methods to minimise the risk of chemical keratopathy must be considered. It is also pertinent to ensure risk factors described above are asked during history-taking as susceptible individuals may need referral to a dermatologist if other suspicious lesions are identified.

Our case poses new questions on the exact pathophysiology of actinic keratosis of the tarsal conjunctiva. It specifically 


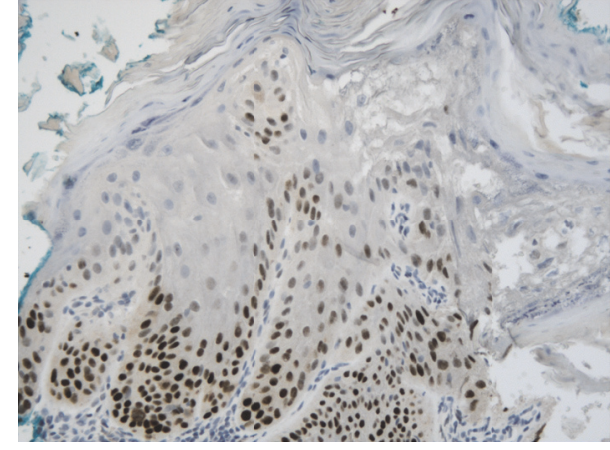

(a) P53 immunohistochemical staining of the lesion demonstrating increased mutational staining and strong, uniform positivity in the basal layers of the lesion. 100x magnification

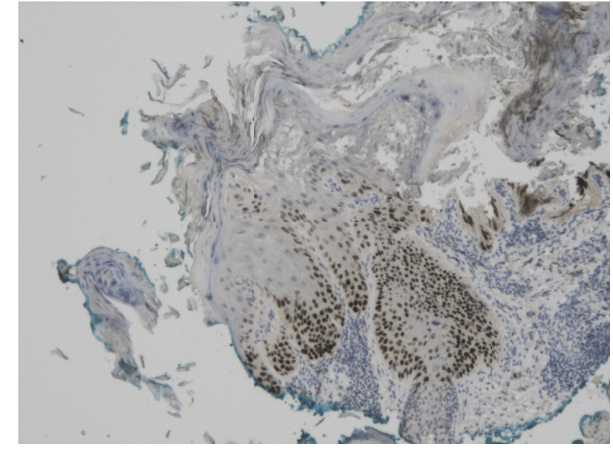

(b) P53 immunohistochemical staining of the lesion demonstrating increased mutational staining and strong, uniform positivity in the basal layers of the lesion. 50x magnification

Figure 3

challenges the general understanding of the exact cause of AK and places the spotlight on the possibility of a contributory genetic component.

\section{Conclusions}

This is an unusual case of AK affecting a non-sun-exposed area and presenting with a leukoplakic appearance. Eversion of the upper eyelid is important to determine the extent of the lesion when it affects the eyelid margin, where possible excisional biopsy of AK is the first-line treatment of choice. New research into chemotaxic agents such as imiquimod has provided the opportunity for less invasive but effective treatment of conjunctival AK. In instances where complete excision is not viable, cryotherapy or imiquimod for conjunctival lesions is a second-line evidence-based therapy.

\section{Consent}

Written informed consent was obtained from this study participant for use of pictures included in this case report.

\section{Disclosure}

This case was previously presented as a poster at European Society of Oculoplastic Surgeons, Athens, 2016.

\section{Conflicts of Interest}

There are no financial interests to declare with respect to either author or conflicts of interest.

\section{References}

[1] J. Shields and C. Shields, Eyelid, Conjunctival, and Orbital Tumours: An Atlas and Textbook, Williams and Wilkins, 2nd edition, 2008, Lippincott.

[2] P. G. Lang Jr., "Management of Actinic Keratoses," Comprehensive Therapy, vol. 29, no. 2-3, pp. 108-114, 2003.
[3] B. Vogelstein, S. Sur, and C. Prives, "p53: The Most Frequently Altered Gene in Human Cancers," Nature Education, vol. 3, no. 9, p. 6, 2010.

[4] L. Naldi, L. Chatenoud, R. Piccitto, P. Colombo, E. B. Placchesi, and C. La Vecchia, "Prevalence of actinic keratoses and associated factors in a representative sample of the italian adult population," JAMA Dermatology, vol. 142, no. 6, pp. S785-S789, 2006.

[5] J. Anwar, D. A. Wrone, A. Kimyai-Asadi, and M. Alam, "The development of actinic keratosis into invasive squamous cell carcinoma: Evidence and evolving classification schemes," Clinics in Dermatology, vol. 22, no. 3, pp. 189-196, 2004.

[6] S. M. Couch and P. L. Custer, "Topical 5-fluorouracil for the treatment of periocular actinic keratosis and low-grade squamous malignancy," Ophthalmic Plastic \& Reconstructive Surgery, vol. 28, no. 3, pp. 181-183, 2012.

[7] A. H. Ross, C. T. C. Kennedy, C. Collins, and R. A. Harrad, "The use of imiquimod in the treatment of periocular tumours," Orbit, vol. 29, no. 2, pp. 83-87, 2010.

[8] M. A. Rowlands, J. N. Giacometti, J. Servat, M. A. Materin, and F. Levin, "Topical Imiquimod in the Treatment of Conjunctival Actinic Keratosis," Ophthalmic Plastic \& Reconstructive Surgery, vol. 33, no. 1, pp. e21-e23, 2017.

[9] B. Mortemousque, F. Leger, C. Brindeau et al., "Actinic keratosis of the conjunctiva. Apropos of a clinical case," Journal Français D’Ophtalmologie, vol. 21, no. 6, pp. 458-461, 1998.

[10] F. D’Hermies, J. Cotinat, C. Elmaleh et al., "Bilateral actinic keratosis of the corneal limbus. Apropos of a case," Journal Français D’Ophtalmologie, vol. 15, 65, no. 1, p. 67, 1992.

[11] J. A. Mauriello Jr, J. Napolatino, and I. McLean, "Actinic keratosis and dysplasia of conjunctiva: A clinicopathological study of 45 cases," Canadian Journal of Ophthalmology, vol. 30, p. 312, 1995.

[12] D. Siegal, S. Tyring, and W. Nahm, “Three-day field treatment with Ingenol Disoxate $0.018 \%$ for actinic keratosis: A phase II trial," The Journal of Clinical and Aesthetic Dermatology, vol. 10, no. 12, pp. 19-26, 2017.

[13] C. Costa, M. Scalvenzi, F. Ayala, G. Fabbrocini, and G. Monfrecola, "How to treat actinic keratosis? An update," Journal of Dermatological Case Reports, vol. 9, no. 2, pp. 29-35, 2015. 
[14] E. Stockfleth, C. Harwood, C. Serra Guillén, T. Larsson, M. L. Østerdal, and T. Skov, "Phase IV head-to-head randomised controlled trial comparing ingenol mebutate $0.015 \%$ with diclofenac sodium $3 \%$ gel for the treatment of actinic keratosis on the face or scalp," British Journal of Dermatology, 2017.

[15] R. Batra, S. Sundararajan, and S. Sandramouli, "Topical diclofenac gel for the management of periocular actinic keratosis," Ophthalmic Plastic \& Reconstructive Surgery, vol. 28, no. 1, pp. 1-3, 2012. 


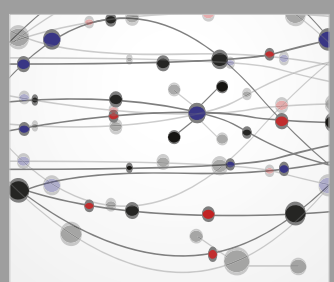

The Scientific World Journal
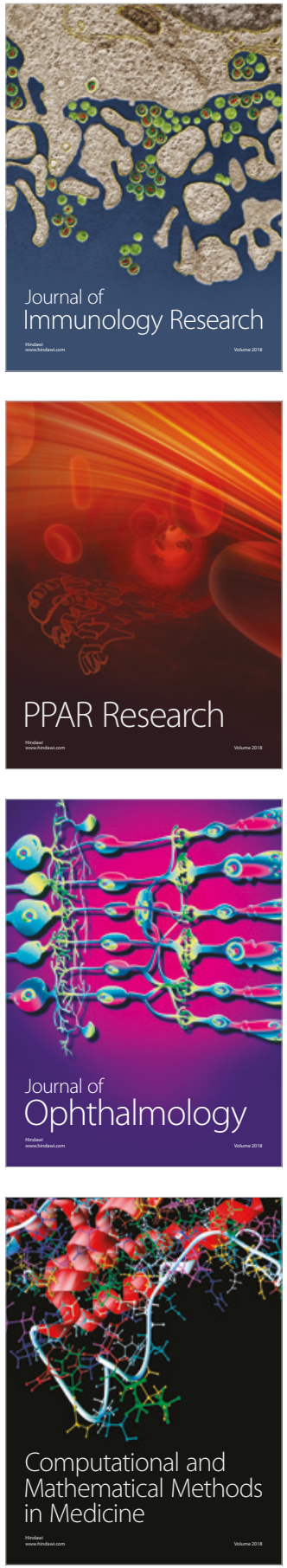

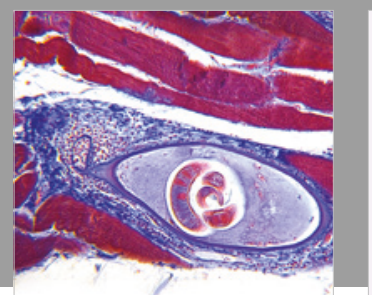

Gastroenterology Research and Practice

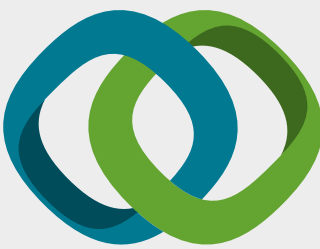

\section{Hindawi}

Submit your manuscripts at

www.hindawi.com
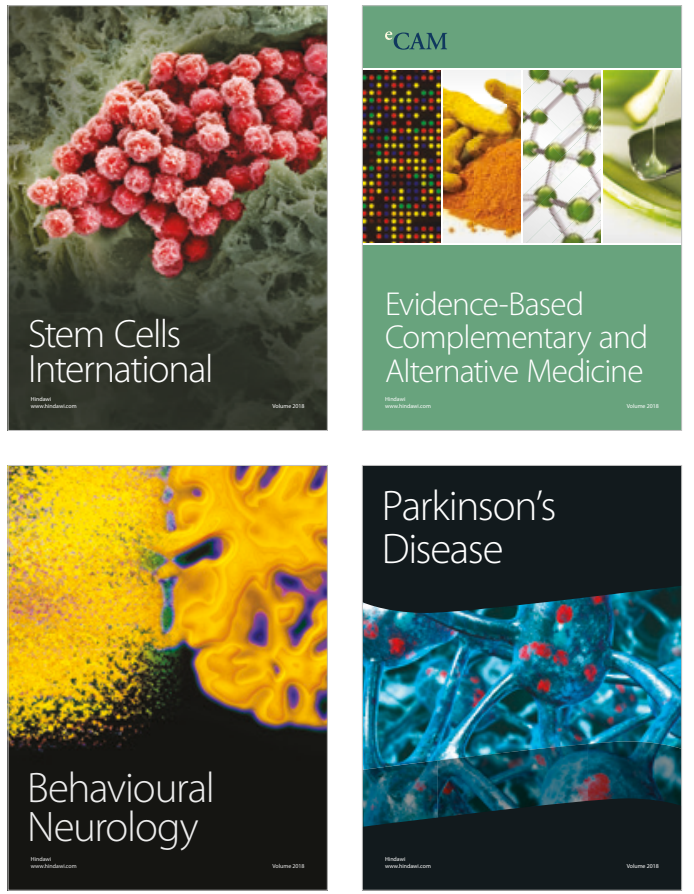

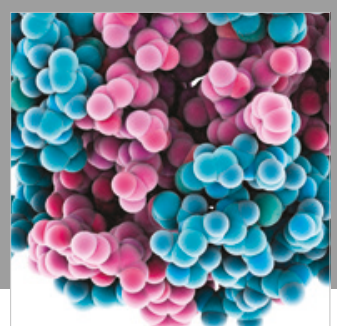

ournal of

Diabetes Research

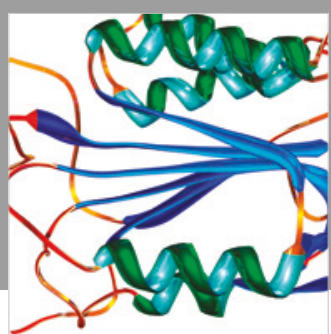

Disease Markers
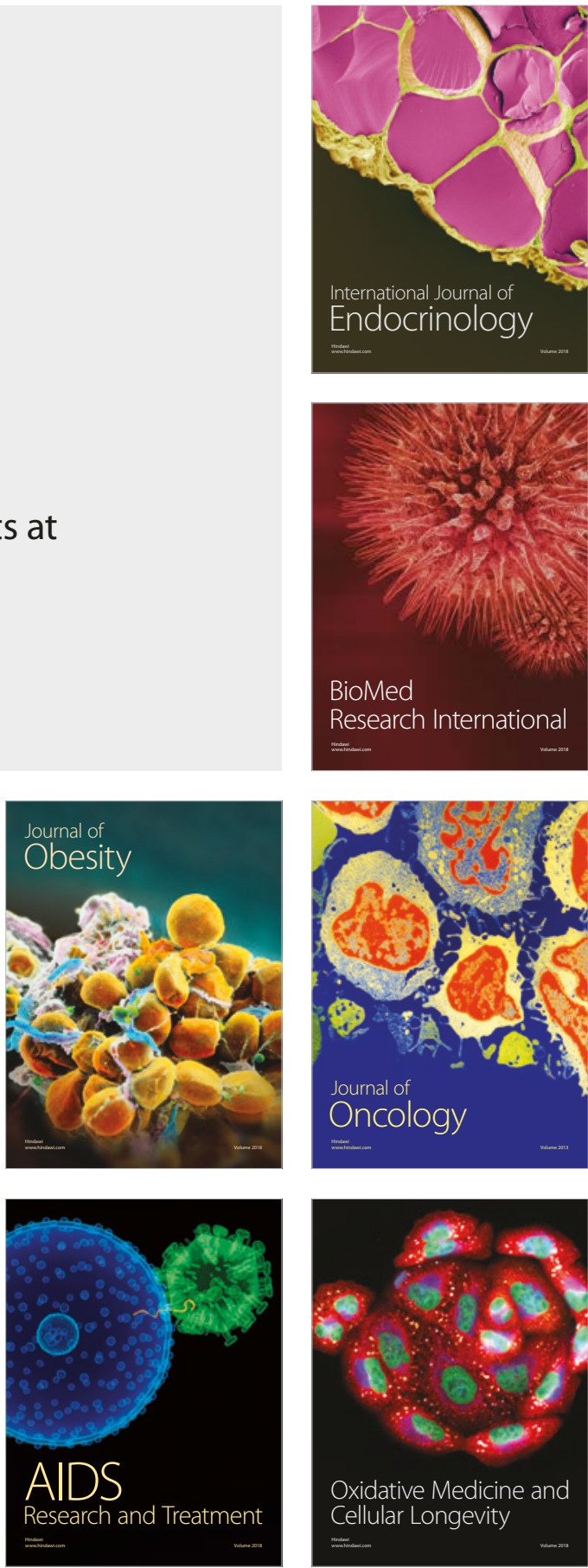